\title{
PERCEPCIONES DE LOS PROFESORES UNIVERSITARIOS: ¿SU ENSEÑANZA ADOPTA UN ENFOQUE CENTRADO EN EL ALUMNO Y SU APRENDIZAJE?
}

\author{
Edurne Chocarro de Luis \\ Universidad de La Rioja \\ Ángel Sobrino Morrás \\ $\mathbf{M}^{\mathrm{a}}$ del Carmen González-Torres \\ Universidad de Navarra
}

\begin{abstract}
RESUMEN: En este artículo se recogen los resultados encontrados al aplicar un cuestionario y una entrevista a profesores universitarios con objeto de conocer algunas de sus creencias pedagógicas pues condicionan su modo de llevar a cabo la enseñanza. La experiencia avala que el Espacio Europeo de Educación Superior (EEES) exige nuevas prácticas docentes, basadas en una enseñanza más centrada en el alumno y su aprendizaje. No obstante este enfoque será más fácil de integrar si los profesores comparten sus principios. Los resultados corroboran esta hipótesis, pues, en general, los docentes apoyan tales premisas aunque todavía perviven ciertas ideas que dificultan su plena puesta en escena, como su control sobre el temario de la asignatura o llevar las riendas del control de la clase.
\end{abstract}

PALABRAS CLAVE: Creencias, profesor, enseñanza.

\section{PROFESSOR EDUCATIONAL BELIEFS: TAKE A LEARNER-CENTERED TEACHING APPROACH?}

\begin{abstract}
This article presents the results obtained from a process based on questionnaires and interviews to lectures, with the aim of learn about their educational beliefs. The interest of this research is the important role that these beliefs play in the usual teaching decisions that professors make. The European Higher Education Area requires new teaching strategies which are summarized in an education model that is focused in the student and his learning. This approach will be more easily implemented if all professors share its principles. The present study concludes that professors do share that
\end{abstract}


principles but certain ideas are still present which make difficult to put the principles entirely in practice.

KEYWORD: Beliefs, lecture, teaching.

Recibido: 17/01/2013

Aceptado: 06/08/2013

\section{INTRODUCCIÓN}

El EEES es ya una realidad: profesores y alumnos universitarios, ilusionados, reticentes o confusos, son partícipes de un nuevo escenario educativo que pretende facilitar un aprendizaje más activo. El alumno adquiere un papel de mayor protagonismo pues el alumno está avocado a más horas de trabajo autónomo. No obstante, esta autonomía no resta, o más bien no debería restar, peso al profesor. En su papel de guía y facilitador debe proporcionar la estructura necesaria para que el alumno, progresivamente, adquiera las herramientas que le permitan ser más responsable de su aprendizaje (Salomon, 1992; Weimer, 2002; Fink, 2003) y con una mayor capacidad para aprender. De acuerdo a Marcelo (2002) esta capacidad se concreta en lo que se ha denominado aprendizaje autorregulado, aspecto que se ha convertido en una de las metas del sistema educativo (Torrano y González-Torres, 2004) y un tema de gran interés actual (De la Fuente, Pichardo, Justicia y Berbén, 2008).

Los distintos modelos instructivos propuestos para la enseñanza de los procesos y las estrategias propios del aprendizaje autorregulado (andamiaje, práctica autorreflexiva, modelado, etc.) se fundamentan en un paradigma denominado enseñanza centrada en el alumno y en el aprendizaje (ECAA) (Chocarro, González-Torres y Sobrino, 2007). Hay 14 principios que configuran el marco teórico de una práctica educativa amparada por tal paradigma y que, a instancias de la APA en 1997, B. McCombs y su equipo, recogió en un documento ya clásico titulado Learner Centered Psychological Principles: Guidelines for School For School Redesign and Reform. Estos principios, como se expone en la tabla 1, están categorizados en torno a cuatro dominios, en alusión a los factores más influyentes en el aprendizaje: factores metacognitivos y cognitivos, motivacionales y afectivos, evolutivos y sociales y diferencias individuales.

Tabla 1. Principios psicológicos de la enseñanza centrada en el alumno. Tomados y traducidos de McCombs y Whisler (2000: 26)

Factores metacognitivos y cognitivos

1. La naturaleza del proceso de aprendizaje: el aprendizaje es más efectivo cuando supone una construcción significativa a partir de las experiencias y el conocimiento previo.

2. Metas del proceso de aprendizaje: el alumno busca crear representaciones congruentes y significativas del conocimiento.

3. La construcción del conocimiento: el aprendiz relaciona significativamente la información nueva con la ya existente. 
4. Pensamiento estratégico: el alumno construye y utiliza una serie de estrategias de pensamiento y razonamiento para alcanzar las metas de aprendizaje.

5. Pensar sobre el pensamiento: el procesamiento de orden superior para la selección y dirección de los procesos mentales potencia el pensamiento crítico y creativo.

6. Contexto de aprendizaje: el aprendizaje depende de factores del ambiente como la cultura, tecnología y la institución escolar.

Factores motivacionales y afectivos

7. Influencias motivacionales sobre el aprendizaje: ¿Cómo y en qué grado la motivación contribuye en el aprendizaje? El estado emocional de la persona, las creencias, el interés y las metas influyen en la motivación.

8. Motivación intrínseca hacia el aprendizaje: la creatividad, el pensamiento de orden superior y la curiosidad facilitan el aprendizaje. Esta motivación se estimula a través de tareas que presentan un grado óptimo de dificultad, se orientan hacia los intereses de los alumnos y les proporciona posibilidad de opción y control.

9. Efectos de la motivación sobre el esfuerzo: la adquisición de un conocimiento y de estrategias complejas requiere esfuerzo y una práctica guiada. Si el alumno no está motivado intrínsicamente hacia el aprendizaje, es difícil que voluntariamente realice ese esfuerzo.

Factores evolutivos y sociales

10. Influencias del desarrollo sobre el aprendizaje: al igual que en el desarrollo personal, existen distintas oportunidades y limitaciones hacia el aprendizaje. Este aprendizaje es más efectivo cuando se tiene en cuenta los distintos niveles de desarrollo físico, intelectual, emocional y social de los alumnos.

11. Influencia social sobre el aprendizaje: la interacción social, las relaciones interpersonales y la comunicación afectan al aprendizaje.

Factores de diferencias individuales

12. Diferencias individuales: Ios alumnos tienen diferentes estrategias, capacidades y perspectivas hacia el aprendizaje que dependen de sus experiencias previas y herencia.

13. Aprendizaje y diversidad: los resultados de aprendizaje son mejores cuando se tienen en cuenta las diferencias lingüísticas, culturales y sociales.

14. Estándares y autoevaluación: establecer niveles adecuados en los retos y evaluar al alumno y su progreso son partes integrantes del proceso de aprendizaje. 
La aplicación al aula del modelo ECAA supone un cambio de mentalidad para alumnos y profesores (Pozo, 2006: 2008), ya que los nuevos roles docentes y discentes requieren que aquellos y estos replanteen sus modos de actuar e incluso, en muchos casos, desaprendan hábitos ya adquiridos. Pozo (2006: 55) menciona que

"la nueva cultura del aprendizaje, está exigiendo de los profesores, pero también de los alumnos, que asuman nuevos modelos o roles funcionales que probablemente entran en conflicto con algunas de esas creencias profundamente arraigadas que constituyen legado cultural y biológico con el que todos los profesores y alumnos llegamos a las aulas".

Los profesores son la clave para lograr este giro en la enseñanza pues, en última instancia, son los artífices de llevarlo a la práctica y de incentivar el aprendizaje autorregulado en sus alumnos. Se convierten pues en la bisagra entre la teoría, lo que debería ser, y la realidad del aula. Sin embargo, en ocasiones sus prácticas distan de las premisas mencionadas ya que sus creencias pedagógicas, que filtran sus decisiones en el aula, proceden de ámbitos academicistas opuestos a los principios mencionados. Entendemos aquí por creencias la disposición a interpretar y actuar de un modo determinado (Samuelowics, 1999).

En este sentido, parece necesario que tomen conciencia de cuáles son estas ideas para reconducir el giro de la enseñanza universitaria hacia el nuevo escenario educativo. Investigaciones europeas recientes señalan que los profesores universitarios estarán más dispuestos a aceptar los principios de la reforma si sus creencias se aproximan a estos (Entwistle, Skinner, Entwistle y Orr, 2000; Postareff, LindblomYlänne y Nevgi, 2007; Reis-Jorge, 2007; Stes, Gijbels y Van Petergen, 2007; Van Driel, Butle y Verloop, 2007; Postareff y Lindblom Ylänne, 2008). También los nuevos modelos de desarrollo profesional docente insisten en trabajar esta cuestión en los programas de formación.

En este marco, los objetivos de este estudio son:

1. Conocer en qué grado los profesores comparten los principios de la ECAA.

2. Analizar aquellas creencias pedagógicas que dificultan la integración al aula de la ECAA.

3. Comparar los resultados obtenidos en un cuestionario y en una entrevista en relación a sus creencias pedagógicas.

La enseñanza es una actividad compleja en la que influyen variables diversas por lo que es imposible que un profesor revele sus opiniones contestando a un único cuestionario. Por ello se utilizó tanto una prueba objetiva como una entrevista para recabar información y, consecuentemente, este trabajo se enmarca en un diseño mixto de investigación, como se explica a continuación.

\section{Método}

El diseño de la investigación se corresponde con el de las investigaciones de métodos mixtos (mixed methods), también conocidas como investigaciones multimétodo (Bericat, 1998; Creswell y Plano Clark, 2007; Creswell, 2009), ya que concurren datos cuantitativos y cualitativos. 
Dentro de este marco de los diseños de investigación mixtos, existen cuatro estrategias para integrar ambos resultados (tabla 2), que se organizan en un continuo en función de los siguientes criterios:

- El peso que tiene el análisis cuantitativo o cualitativo.

— El momento en que se realizan uno u otro análisis y su interpretación.

Tabla 2. Estrategias de métodos mixtos de investigación. Tomada y traducida de Cresswell y Plano Clark (2007: 85)

\begin{tabular}{cccc}
\hline Diseños & Integración & Tiempo & Peso \\
\hline Triangulación & $\begin{array}{c}\text { Se fusionan los datos } \\
\text { durante la interpretación y } \\
\text { el análisis }\end{array}$ & $\begin{array}{c}\text { Cualitativo y } \\
\text { cuantitativo al } \\
\text { mismo tiempo }\end{array}$ & Equitativo \\
\hline $\begin{array}{c}\text { Complementario } \\
(\text { EMBED })\end{array}$ & $\begin{array}{c}\text { Se integran un tipo de datos } \\
\text { en un diseño de otro tipo de } \\
\text { datos }\end{array}$ & $\begin{array}{c}\text { Simultáneo o } \\
\text { secuencial }\end{array}$ & Desigual \\
\hline Explicativo & $\begin{array}{c}\text { Se conectan los datos entre } \\
\text { las dos fases }\end{array}$ & Secuencial & Cuantitativo \\
\hline Exploratorio & $\begin{array}{c}\text { Se conectan los datos entre } \\
\text { las dos fases }\end{array}$ & Secuencial & Cualitativo \\
\hline
\end{tabular}

El diseño elegido es el de complementación: "existe complementación cuando, en el marco de un mismo estudio, se obtienen dos imágenes, una procedente de métodos de orientación cuantitativa y otros de métodos de orientación cualitativa. Así, obteniendo esta doble y diferenciada visión de los hechos complementamos nuestro conocimiento sobre los mismos" (Bericat, 1998: 37). No obstante, el peso de los datos obtenidos de una y otra imagen de procedencia es diferente, y unos se acoplan a otros. En nuestro este trabajo, los cualitativos se añaden al estudio tras ya tener los cuantitativos que, a su vez, tienen más peso en esta investigación.

\section{Instrumentos}

En esta investigación, se utilizó:

1. El cuestionario Assessment Learner Centered Practices (ALCP), en su versión para el ámbito universitario desarrollado por McCombs y Pierce en 1999.

2. Una entrevista de 6 preguntas, aplicada a profesores.

\section{Assessment Learner Centered Practices (ALCP)}

La utilización del ALCP se debe porque: a) tiene una versión propia para el ámbito universitario, b) se basa en los mismos principios establecidos por la APA como referentes de una enseñanza activa y participativa y c) tiene dos versiones, una para docentes y otra para discentes, posibilitando contrastar sus resultados.

Se aplicaron ambas versiones pero aquí sólo se presentan las recogidas por los profesores. 
El cuestionario de los profesores consta de 105 ítems dividido en cinco áreas que se detallan en la Tabla 3, como así también sus subescalas, con el número de ítems correspondiente indicados en la columna de la izquierda. Cada uno de los ítems se contesta en una escala de 4 puntos (donde 1 es totalmente en desacuerdo y 4 totalmente de acuerdo). En la tabla se destacan también, el índice de fiabilidad encontrado en las subescalas.

Tabla 3. Escalas y factores del ALCP de la versión para profesores

\begin{tabular}{|c|c|c|c|}
\hline $\begin{array}{c}\text { Áreas objeto de } \\
\text { análisis }\end{array}$ & Subescalas que configuran cada área & $\begin{array}{l}\text { Número } \\
\text { de ítem }\end{array}$ & $r_{x x}$ \\
\hline \multirow{3}{*}{$\begin{array}{c}\text { Creencias y } \\
\text { percepciones acerca } \\
\text { de los alumnos, el } \\
\text { aprendizaje y la } \\
\text { enseñanza }\end{array}$} & $\begin{array}{l}\text { 1. Sobre una enseñanza centrada en el } \\
\text { alumno y su aprendizaje }\end{array}$ & 14 & .80 \\
\hline & $\begin{array}{l}\text { 2. En torno al alumno que dificultan la } \\
\text { enseñanza centrada en el alumno }\end{array}$ & 8 & .67 \\
\hline & $\begin{array}{l}\text { 3. En torno a variables del proceso } \\
\text { de enseñanza-aprendizaje que lo } \\
\text { dificultan }\end{array}$ & 8 & .52 \\
\hline \multirow{5}{*}{$\begin{array}{c}\text { Características } \\
\text { de la enseñanza } \\
\text { efectiva (acorde con } \\
\text { los principios de } \\
\text { enseñanza centrada } \\
\text { en el alumno) }\end{array}$} & $\begin{array}{l}\text { 1. Adaptación de la enseñanza a las } \\
\text { necesidades de aprendizaje de los } \\
\text { alumnos }\end{array}$ & 10 & .68 \\
\hline & $\begin{array}{l}\text { 2. Establecimiento de relaciones sociales } \\
\text { positivas }\end{array}$ & 10 & .71 \\
\hline & $\begin{array}{l}\text { 3. Facilitar el proceso de ap. y la } \\
\text { enseñanza y el uso de estrategias de } \\
\text { aprendizaje }\end{array}$ & 10 & .78 \\
\hline & $\begin{array}{l}\text { 4. Incentivar el reto, la toma de decisiones, } \\
\text { elecciones y la responsabilidad } \\
\text { personal de los alumnos }\end{array}$ & 6 & .55 \\
\hline & $\begin{array}{l}\text { 5. Responder a las necesidades sociales y } \\
\text { personales de los alumnos }\end{array}$ & 6 & .80 \\
\hline $\begin{array}{l}\text { Variables en relación } \\
\text { a la motivación }\end{array}$ & Autoeficacia del profesorado & 6 & \\
\hline Práctica reflexiva & Práctica reflexiva & 14 & \\
\hline Demográficas & Género, curso, materia, grado & 13 & \\
\hline
\end{tabular}

La primera, Creencias de los profesores sobre la enseñanza, el aprendizaje y los alumnos, constituye un cuestionario en cierta medida autónomo puesto que puede utilizarse de manera independiente al resto, como autorreflexión sobre las propias creencias pedagógicas. De ahí que los datos recabados de esta escala son los presentados en este trabajo y que responden al objetivo de investigación.

Esta escala consta de las siguientes subescalas:

1. Creencias en torno a una enseñanza centrada en el alumno y su aprendizaje. En esta escala concurren ítems que recogen las ideas principales de la ECAA.

2. En torno a variables referidas al alumno que dificultan la puesta en práctica de una ECAA. 
3. En relación a variables del proceso de enseñanza que dificultan la puesta en práctica de una ECAA. Esta subescala y la anterior hace referencia a ideas opuestas a una ECAA y, por tanto, su interpretación se hace a la inversa.

Los profesores respondieron al ALCP vía on-line (se envió un mail invitándoles a participar donde se especificaba el enlace que accede al cuestionario).

\section{Entrevista}

La entrevista empleada se confeccionó a partir de la revisión de otras utilizadas en este campo de estudio (Hativa, 1997; Ho, 2000; Postareff y Lindblom-Ylänne, 2008). Consta de seis preguntas que indicamos a continuación.

1. Supongo que al inicio del curso académico revisa su programación, o planifica el transcurso del curso. Así establece el temario, actividades, etc. La pregunta que me gustaría formular es: cuando lleva a cabo su programación, ¿qué pretende que logren sus alumnos al finalizar el curso?

2. De todo ello, ¿qué considera que es lo principal como profesor?

3. El transcurso de las clases es distinto de unas a otras e incluso la sesión dedicada a un mismo contenido es distinta de un año a otro. Así que seguro que en ocasiones, ha finalizado la clase satisfecho del resultado o del desarrollo de la misma. ¿Qué variables o cuáles son los elementos que le llevan a pensarlo? ¿Qué cree que ha sido lo que le ha hecho salir contento de la clase?

4. Cada vez se habla más de que el alumno debe asumir un papel más activo en el proceso de enseñanza y un mayor protagonismo ¿opina que está preparado?

5. La universidad camina hacia modelos de enseñanza más activos ¿lo ve fácilmente viable o considera que hay variables que influyen en su puesta en práctica?

6. ¿Cuál ha sido su principal fuente de información para afrontar la tarea de enseñar?

7. En este trabajo, presentamos los resultados obtenidos en las tres primeras que responden a los tres objetivos de investigación mencionados en la introducción.

\section{Muestra}

Detallamos, por un lado, la muestra objeto de aplicación del cuestionario ALCP y, por otro, los profesores que accedieron a ser entrevistados.

Muestra a la que se aplicó el ALCP

La muestra está configurada por los 64 docentes, 30 mujeres y 34 hombres, que voluntariamente respondieron al cuestionario tras enviarlo vía mail a todo el profesorado de la Universidad de Navarra, con sede en Pamplona. Respecto a sus áreas de enseñanza, el número de profesores fue:

- Ciencias de la Salud (enfermería y medicina)

- Facultad de Ciencias

- Ciencias Sociales

- Escuela de Arquitectura 
Muestra a la que aplicó la entrevista

La muestra, de carácter incidental, estuvo forma por 9 de los profesores que habían respondido al cuestionario y mostraron su interés por continuar tomando parte en el estudio. De ellos, ocho pertenecen a la Facultad de Ciencias y uno a la Facultad de Derecho.

\section{Análisis de datos}

De acuerdo a lo antes mencionado, se utilizó la estrategia de la complementación, ya que los datos procedentes de la entrevista sirvieron de apoyo para la interpretación de los obtenidos del cuestionario, integrándose a estos. Por tanto, se otorgó más peso al análisis cuantitativo, siendo el cualitativo un apoyo, ya que el ALCP es nuestra principal herramienta. Además, el análisis no fue simultáneo: el cuantitativo se llevó a cabo en primer lugar.

\section{Análisis cuantitativo}

Los datos recabados de la aplicación del ALCP en alumnos y profesores se han analizado con el SPSS versión 15.0. Se ha realizado un análisis descriptivo tanto de cada una de las escalas del ALCP y sus correspondientes subescalas, como de sus ítems.

\section{Análisis cualitativo: análisis de contenido}

Una vez transcritas las 9 entrevistas, se analizaron utilizando la técnica del análisis de contenido, "una técnica de investigación para la descripción objetiva, sistemática y cuantitativa del contenido manifiesto de las comunicaciones y que tiene como objetivo interpretarlas" (Barelson, 1952: 501).

Los dos procesos más importantes del análisis de contenido son la codificación y la categorización. La codificación corresponde a una transformación - efectuada según reglas precisas - de los datos del texto (Bardin, 1986: 78). Estas reglas son la unidad de registro ${ }^{1}$ (el contenido enunciado) y la enumeración de esos datos (la manera de registrar o contar lo enunciado) ${ }^{2}$. En este trabajo se ha adoptado el tema como unidad de registro en cuanto que se descompone los textos en enunciados que aportan información relevante para esta investigación y, como criterio para enumerarla, su frecuencia de aparición en el conjunto de los textos.

En cuanto a la categorización (análisis categorial), el otro de los dos procesos, se refiere a la clasificación de las unidades de registro. Es obvio que los procesos de codificación y de categorización se intercalan pues están relacionados. Y de ahí que, en este trabajo, el tema es también la misma categoría. Barelson (1952) propone como otras posibles categorías: orientación (positiva o negativa), valores o métodos.

1. La unidad de registro es la unidad de significación establecida que se utilizará con miras a la categorización y al recuento de frecuencia. Estas unidades o segmentos pueden ser, de acuerdo a Barelson (1952), la palabra, el tema, el ítem o la persona.

2. La enumeración, la manera de contar esas unidades de registro, puede llevarse teniendo en cuenta su presencia o ausencia así como su frecuencia, orden (momento de aparición) o contingencia. 
Para establecer los temas como categorías, primeramente se realizó una lectura superficial de las entrevistas ya transcritas pues, de este modo, obteníamos una visión panorámica y general de la información en su conjunto que nos permitía establecer posibles líneas para comenzar a categorizar. No obstante, este proceso está ya contaminado, pues las preguntas fijan, aunque no es previsible por su carácter abierto, los temas que los profesores pueden abordar. Por contra, las subcategorías concretas se establecieron tras analizar los resultados.

A continuación se enumeran las cuestiones de estudio (categorías) y, en paréntesis, se indican las preguntas en las que, sobre todo, los profesores hablaron de ellas:

- objetivos de aprendizaje (pregunta 1 y 2)

- características de una buena práctica (pregunta 3),

- participación del alumno (pregunta 4).

\section{Resultados}

\section{Fase cuantitativa}

Se presentan, en primer lugar, las medias obtenidas en la Escala I recabadas del total de la muestra de profesores $(n=64)$ en respuesta al primer objetivo, y se recuerda que las puntuaciones pueden obtener un máximo de 4 puntos. Como se ha explicado, esta Escala consta a su vez de tres subescalas (que se enumerarán como 1,2 y 3) y se presentarán también las puntuaciones dadas a algunos de sus respectivos ítems que responden al segundo objetivo de investigación. En segundo lugar, se desglosará el análisis de contenido para las categorías: Objetivos de aprendizaje, Participación del alumno y Características de una buena práctica para comparar los resultados obtenidos en un cuestionario y en una entrevista en relación a sus creencias pedagógicas.

Así pues, en la tabla 4 se presentan los datos obtenidos en la aplicación a los 64 profesores en la Escala I:

Tabla 4. Medias y desviación típica de las subescalas de la escala Creencias y percepciones acerca de los alumnos, el aprendizaje y la enseñanza $(n=64)$

\begin{tabular}{llcc}
\hline \multicolumn{1}{c}{ SUBESCALAS } & MEDIA & SD \\
\hline 1. & De una enseñanza centrada en el alumno & 3.03 & 0,37 \\
\hline $\begin{array}{l}\text { 2. } \\
\text { aprendizaje que lo dificultan }\end{array}$ & 2.68 & 0,45 \\
\hline $\begin{array}{l}\text { Sobre el alumno que dificultan la enseñanza centrada } \\
\text { en el alumno }\end{array}$ & 2.41 & 0,42 \\
\hline
\end{tabular}

3. Las preguntas 1 y 2 se analizaron de modo conjunto bajo el rótulo Objetivos de aprendizaje, ya que estaban estrechamente relacionadas: la primera interroga sobre las finalidad pretendidas por el profesor con su enseñanza y la segunda a la función que el docente se asigna en relación a esas finalidades. 
En general, de acuerdo al valor medio de la primera de las escalas, los profesores tienden a mostrar su adhesión a los principios de la enseñanza centrada en los alumnos/aprendizaje, lo cual pone de relieve que son conscientes de su labor educativa y que no se ven a sí mismos, como podría preverse dada la tradicional cultura academicista recibida, simplemente como "expertos" que deben impartir un contenido. No se podría decir esto si, a la inversa, las puntuaciones más altas hubieran sido las de las subescalas 2 y 3 que hacen referencia a ideas que dificultan la integración de una enseñanza centrada en el alumno y su aprendizaje.

A continuación se indican algunos de los ítems de cada una de las subescalas destacables tanto por su valoración global como por su significado e implicación en la práctica. Así, puede verse con más claridad cuáles son las creencias pedagógicas que sugieren que estos docentes no se decanten abiertamente por un modelo ECAA. Se recuerda que el recorrido de las respuestas del cuestionario oscila de 1 a 4 puntos.

En respuesta al segundo objetivo de investigación (conocer cuáles son las creencias pedagógicas que dificultan una ECAA) y por lo que respecta a la primera de las subescalas (tabla 5), señalamos que los profesores estiman positivamente la relación e interacción con los estudiantes, característica importante sobre todo de cara a los nuevos aires de reforma donde la tutoría toma más peso, pero siempre en relación a temas sobre el aprendizaje, y destacando que tampoco la consideran lo más crucial. Esto es, aunque lo estiman influyente para crear un clima óptimo de aprendizaje no lo perciben como un requisito imprescindible.

Tabla 5. Ítems destacados de la escala Creencias y percepciones de una enseñanza centrada en el alumno

\begin{tabular}{lccc}
\hline \multicolumn{1}{c}{ ÍTEMS } & M & SD \\
\hline $\begin{array}{l}\text { Escuchar a los alumnos con interés les ayuda a resolver sus problemas } \\
\begin{array}{l}\text { Los estudiantes rinden más cuando los profesores les animan a expresar } \\
\text { sus opiniones y sentimientos }\end{array}\end{array}$ & 3.39 & .53 \\
$\begin{array}{l}\text { Los estudiantes se motivarán más si los docentes muestran interés por } \\
\text { conocerlos }\end{array}$ & 3.34 & .69 \\
$\begin{array}{l}\text { Ser una figura autoritaria y distante resulta menos efectivo para el } \\
\text { aprendizaje de mis alumnos que mostrarme cercano }\end{array}$ & 3.16 & .96 \\
$\begin{array}{l}\text { Los estudiantes demuestran un mayor respeto por aquellos docentes a } \\
\text { los que pueden ver no sólo como su profesor sino como una persona } \\
\text { cercana con las que se pueden relacionar }\end{array}$ & & \\
$\begin{array}{l}\text { Dedicar tiempo a establecer relación con mis alumnos es el factor más } \\
\text { importante para su aprendizaje y motivación }\end{array}$ & 2.80 & \\
$\begin{array}{l}\text { Ayudar a los estudiantes a entender cómo sus ideas sobre sí mismos } \\
\text { influyen en su aprendizaje es tan importe como trabajar en sus aptitudes } \\
\text { académicas }\end{array}$ & 2.69 & \\
$\begin{array}{l}\text { Una de las cosas más importantes que puedo enseñar a mis alumnos es } \\
\text { aprender a hacer frente a las presiones propias del mundo laboral }\end{array}$ & 2.77 & & \\
\hline
\end{tabular}


Aún conscientes de que los profesores de la muestra son docentes que voluntariamente decidieron participar en el estudio, interesa, más que las puntuaciones, destacar que los cinco primeros ítems de la tabla recogen ideas que evocan la imagen de un profesor más próximo al rol de facilitador del aprendizaje y no solo de transmisor.

Sin embargo, y respecto a puntuaciones de los ítems de la subescala 2, vemos que todavía tienen peso ciertas ideas que pueden limitar un tanto la puesta en práctica del rol de facilitador como se refleja en la tabla 6.

Tabla 6. Ítems destacados de la escala Creencias en relación al proceso de enseñanza y aprendizaje que lo dificultan $(n=64)$

\begin{tabular}{lcc}
\hline \multicolumn{1}{c}{ ÍTEMS DESTACADOS } & M & SD \\
\hline $\begin{array}{l}\text { Necesito llevar las riendas sobre el contenido y el proceso de aprendizaje } \\
\text { para lograr altos niveles de enseñanza y aprendizaje }\end{array}$ & 2.97 & .75 \\
$\begin{array}{l}\text { Como profesor, soy responsable de lo que aprenden mis alumnos y de } \\
\text { cómo lo aprenden }\end{array}$ & 3.25 & .69 \\
$\begin{array}{l}\text { Dominar mi materia es el requisito más importante para ser un buen profesor } \\
\text { Dominar mi materia lo mejor posible es lo más importante para }\end{array}$ & 2.78 & .76 \\
$\begin{array}{l}\text { contribuir al aprendizaje de mis alumnos } \\
\begin{array}{l}\text { Tengo que mostrar que soy la autoridad en mi campo y no puedo } \\
\text { permitirme cometer errores ante mis alumnos }\end{array}\end{array}$ & 1.95 & .73 \\
\hline
\end{tabular}

Estos ítems se interpretan a la inversa, esto es, su resultado es mas favorable, desde el punto de vista de la integración de los principios antes expuestos, cuanto más baja sea su puntuación, ya que al formar parte de la subescala 3 están enunciados de modo negativo. Lo mismo sucede con la subescala 2.

Cabe puntualizar que puede que los profesores interpretaran los dos primeros ítems desde el punto de vista de su responsabilidad frente a la enseñanza como docentes. De esta subescala, sobre todo, y en línea con nuestra tradición, cabe señalar que los profesores priman el contenido y su conocimiento del mismo pero no olvidan que son personas y es importante mostrar su cercanía, como se ha visto ("Tengo que mostrar que soy la autoridad en mi campo y no puedo permitirme cometer errores ante mis alumnos").

Algunos de los datos más destacados de la subescala 3 (tabla 7) son los siguientes (téngase en cuenta que esta escala también se interpreta a la inversa):

Tabla 7. Ítems destacados de la escala Creencias y percepciones sobre el alumno que dificultan la enseñanza

\begin{tabular}{lcc}
\hline \multicolumn{1}{c}{ ÍTEMS DESTACADOS } & M & SD \\
\hline No importa lo que haga, a determinados alumnos no conseguiré motivar & 2.36 & .91 \\
$\begin{array}{l}\text { Poco puedo hacer para motivar a los alumnos que se niegan a estudiar } \\
\text { En la Universidad, ya es tarde para ayudarles a aprender las estrategias }\end{array}$ & 2.03 & .87 \\
básicas de aprendizaje & 1.64 & .84
\end{tabular}




\begin{tabular}{lccc}
\hline \multicolumn{1}{c}{ ÍTEMS DESTACADOS } & M & SD \\
\hline $\begin{array}{l}\text { La inteligencia y la capacidad están rígidamente fijadas y algunos } \\
\text { estudiantes no tienen lo necesario para rendir bien en la Universidad }\end{array}$ & 2.19 & .87 \\
$\begin{array}{l}\text { No se puede esperar que los docentes en la Universidad trabajen con los } \\
\text { alumnos que sistemáticamente ocasionan problemas en el aula }\end{array}$ & 2.25 & .83 \\
$\begin{array}{l}\text { Algunos estudiantes todavía no dominan las estrategias básicas de } \\
\text { lectura y estudio necesarias para el trabajo en la Universidad }\end{array}$ & 3.48 & .66 \\
$\begin{array}{l}\text { Muchos alumnos todavía no tienen la madurez necesaria y, de hecho, } \\
\text { esperan que se les "mime" }\end{array}$ & 2.92 & .87 \\
\hline
\end{tabular}

Es positivo que los docentes crean, en primer lugar, en su capacidad para motivar y facilitar el aprendizaje y, en segundo lugar, consideren que el alumno aún puede adquirir las estrategias durante su paso por la universidad. Estas notas toman más peso cuando, por otra parte, no perciben al alumnado universitario muy preparado para asumir la responsabilidad en su aprendizaje que demanda Bolonia como lo indican las puntuaciones de los últimos ítems de la tabla.

En definitiva, una vez examinados los resultados podemos decir que los profesores se adhieren a los principios de la enseñanza centrada en el alumno aunque todavía persisten ciertos aspectos por mejorar como así se constató en las entrevistas que pasamos a analizar a continuación.

\section{Fase cualitativa}

En este apartado se desglosará el análisis de contenido de las siguientes categorías establecidas: Objetivos de aprendizaje, Participación del alumno y Características de una buena práctica.

En cuanto a la primera de las categorías: OBJETIVOS DE APRENDIZAJE se realizó siguiendo la propuesta de Fink (2003) y los profesores hicieron mención a cada una de las dimensiones de aprendizaje establecidas por este autor:

Conocimiento funcional: 3 profesores utilizaron el verbo comprender para explicar sus metas de aprendizaje o su función docente para con sus alumnos: "hacer comprender" (N1), "lo primero es que comprendan las asignaturas" (N3), "comprendan la asignatura" (N8).

Aplicación: 4 profesores pronunciaron el verbo aplicar: "que se den cuenta de qué aspectos pueden tener más aplicación para su futuro ejercicio profesional [...] que vean la utilidad de esa materia para su ejercicio profesional" (N2), "sean capaces de tomar todos sus conocimientos básicos para enfrentarse a un problema real" (N6) y "comprendan la asignatura, apliquen sus conocimientos" (N8), "pretendo que adquieran unos conocimientos, que sepan aplicarlos [...]" (N9).

Integración: 3 profesores hicieron mención de la importancia de relacionar y comparar los contenidos totales de la asignatura mencionando ideas como: "enseñar a [...] y a ver las relaciones entre las cosas" (N3), "en primer lugar que los alumnos lleven unas ideas claras sobre los conceptos claves y después pretendo que no solo los memoricen sino que aprendan a relacionarlos y que aprendan a ver qué aspectos 
de un tema están relacionados con otros o qué tengan en cuenta que van a estar relacionados" (N2), "tengan una perspectiva global de cómo funciona [...]" (N6).

Dimensión humana: 3 profesores hacen referencia a la misma; 1 profesor propuso entre sus objetivos que: "mi principal función es formar no solo académicamente a esos alumnos sino de manera integral, yo pretendo que ellos sean responsables de su tarea como estudiantes [...]" (N9). Otro mencionó: "hay dos objetivos: prepararles en lo técnico [...] y enseñarles a convivir" (N7). Y por último, hay quien afirmó: "siempre me ha parecido importante el que aprendan o adquieran una serie de actitudes: hablar en público [...]" (N4).

Cuidado: Conviene apuntar que en este caso, "cuidado" hace referencia a aspectos como preocupación o interés, su opuesto sería indiferencia, y no, como podía parecer a atender, vigilar o incluso mimar a los alumnos. 3 profesores nos expresaron su preocupación por incentivar la curiosidad de los estudiantes que detallamos debido a su particularidad:"lograr que la asignatura les llegue a gustar tanto como me gusta a mi" (N2), "mis alumnos comprendan la asignatura, apliquen sus conocimientos, no sé sintetizarla, creo que mi principal objetivo es al finalizar el curso es que los alumnos se sientan atraídos por [...] porque ven en ello algo que no veían antes" (N8). En respuesta a su función como docente, hubo quien dijo: "mostrarles entusiasmo por aprender" (N9).

Aprender a aprender: 3 fueron los profesores que resaltaron esta dimensión. Una de las intervenciones: "enseñar a pensar y a ver" (N3), otra fue: "intentar que los alumnos participen y en la medida que lo hacen, Ilevan a cabo tareas cómo razonar, analizar, etc." (N5) y la última en relación a esta dimensión fue: "pretendo que desarrollen el espíritu crítico y el razonamiento entonces mi tarea es mostrarle entusiasmo por aprender" (N9).

Como puede verse, los profesores hacen hincapié en incentivar las estrategias del aprender a aprender $y$, sobre todo, en resaltar la funcionalidad del contenido objeto de su enseñanza, que por otra parte, excepto cuatro profesores, lo etiquetaron de esencial o clave: "conceptos básicos mínimos" (N1), "se lleven unas ideas claras sobre los conceptos clave" (N2), "contenidos esenciales" (N4) "hacer comprender los fundamentos de la asignatura" (N5), "conocimientos básicos" (N6).

Los profesores son conscientes de la importancia de seleccionar los contenidos fundamentales, que será el conocimiento base que los alumnos necesitarán.

Una idea central que hemos destacado es el cambio en el punto de mira desde el contenido al aprendizaje del alumno. Podemos decir que la clave está en cuidar lo que el profesor hace y da en clase pero "contando con" el alumno durante el proceso de enseñanza (percatándose de que entiende, comprende y participa en clase). Y así lo reflejaron los profesores en las entrevistas como refleja el análisis de la categoría Buena práctica.

BUENA PRÁCTICA, tiene a su vez las siguientes subcategorías: Ilegar al alumno, cubrir el contenido, claridad y coherencia en la exposición.

El $100 \%$ respondió a la subcategoría Llegar al alumno y dijeron que era el indicador más fiable para ponderar su buen hacer como docente. Más en concreto 4 de ellos utilizaron la expresión "las caras de los alumnos" como la principal de las evidencias para pensar que el desarrollo y el resultado pretendido con esa clase ha sido satisfactorio: "la expresión de sus caras, te das cuenta enseguida de si te siguen o si 
se han perdido" (N1), "pues mira, es una percepción un poco difícil de definir pero cuando tu estás hablando tu lo notas en cómo te miran, en la cara que ponen" (N3), "las caras de los alumnos, si las caras de los alumnos son de interés de mmm, ya está, la clase ha salido bien" (N7), "yo me fijo bastante en la cara de los alumnos y en las dudas que hay a lo largo de la clase"(N9).

En esta línea, uno de los encuestados señaló que aquello que le hace sentirse satisfecho tras una clase es "la elaboración del momento". Este profesor consideró que a pesar de que uno se prepara y organiza la clase previamente, siempre su desarrollo supone un proceso de elaboración: "no cabe duda de que cuando estás dando la clase, hay una especie de pensamiento a la vez que estás hablando, qué atentos, cómo le está gustando lo que les estoy diciendo etc. Y eso claro, a ti también, te hace crecerte, supongo" (N8). Este profesor habla de su proceso de elaboración pero el alumno es el responsable último del desarrollo y desenlace de tal elaboración, por lo que replantea su enseñanza en función de cómo evoluciona el transcurso de la clase.

Dos profesoras, mencionaron las otras dos subcategorías: Cubrir el contenido y Claridad y coherencia en la exposición con testimonios como: "entonces si consigo dar el contenido programado de esa hora de clase y además me doy cuenta de que los alumnos la han seguido, pues eso es lo ideal" (N1), "para empezar tener yo misma claras las ideas, haberlas expuesto con cierto orden y coherencia, eh, eh, darme cuenta de que los alumnos sí las han captado" (N2).

En resumen, los profesores manifestaron, por un lado, la necesidad de apreciar los objetivos que se persiguen en una enseñanza basada en competencias: aplicación, integración del conocimiento, etc. y, por otro, su preocupación por percatarse de que sus alumnos están aprendiendo.

Al analizar la pregunta 44, los docentes manifestaron que los alumnos no están preparados para asumir las riendas de su aprendizaje. Su análisis se categorizó bajo el rótulo:

PARTICIPACIÓN DEL ALUMNO, que se divide en Falta de estrategias de aprendizaje y de motivación, y Cambio de mentalidad. 5 profesores resaltaron la primera de éstas con afirmaciones como: "hombre pues yo creo que están un poco verdes porque a los alumnos que tenemos les acostumbramos, por ejemplo, en general, a darles unos apuntes en clase y que prácticamente eso sea el contenido que tienen que estudiar" (N1), "cuando se ven en el terreno práctico, les resulta difícil extrapolar lo que han estudiado en teoría a la práctica" (N2), "está acostumbrados a: tú te estudias esto de aquí a aquí, están acostumbrado a apuntes"(N3), "hay un alta desmotivación, entre el alumnado habrá sólo un 30 o 40 \% que podrán seguir porque harán los que se les dice y están motivados pero un elevado número de alumnos [...]" (N5), "no saben a veces dónde están, tienen un concepto erróneo y a veces indisciplinado. En el aula la disposición es increíble, un número importante de alumnos que asiste a las clases, y no tiene un especial interés por tomar unos buenos apuntes" (N6).

De estas aportaciones también se desprende que los alumnos, como los profesores, necesitan cambiar la mentalidad sobre su modo de entender el proceso de ense-

4. "Cada vez más se habla que el alumno debe asumir un papel más activo en el proceso de enseñanza y un mayor protagonismo ¿opina que está preparado?" 
ñanza-aprendizaje. En cuanto a los primeros, 2 docentes nombraron la subcategoría Cambio de mentalidad: "están acostumbrados, en general, a basarse en los apuntes. Entonces claro, ahora tenemos que cambiar todos de mentalidad" (N1), "indudablemente el alumno tiene que cambiar totalmente la filosofía" (N3).

En cuanto a los profesores, de estas últimas intervenciones citadas, puede leerse entre líneas que los alumnos están acostumbrados a una enseñanza directiva y los encuestados se quejan de ello. Por consiguiente, en relación al objetivo que estamos estudiando, conocer el grado en que los profesores comparten los principios de la enseñanza centrada en el alumno, podemos intuir que todavía queda camino que recorrer. En una de las entrevistas una profesora, al hilo del tema de la escasa capacidad del alumno para asumir una mayor autonomía, resaltó "o sea, en parte es nuestra culpa" (N1). Sin embargo, otro profesor mencionó: "a mi me parece que, antes de que yo me enterara de lo que era Bolonia, yo ya lo estaba aplicando" (N7). A lo anteriormente dicho se suma que 4 de los encuestados indicaron que los profesores necesitan un cambio de actitud frente a la enseñanza para abordar los nuevos retos educativos.

En resumen, aunque los profesores ponen el acento en el trabajo del alumno, sus revelaciones indican que se encuentran a caballo entre un modelo de transmisión y otro que acentúa más el papel del alumno, no siguen una enseñanza plenamente centrada en el alumno pues todavía priman la relevancia de los apuntes como pilar fundamental de su enseñanza. Es conclusivo que perciben a sus alumnos poco maduros o preparados para asumir cierta responsabilidad en su aprendizaje pero creen en la posibilidad de solventarlo en cierto modo durante su permanencia en la universidad.

\section{Discusıón}

Este estudio multimétodo se llevó a cabo con el objeto de recoger las impresiones de profesores sobre los principios del proceso de enseñanza-aprendizaje que fundamentan el actual reto del EEES. En este marco es una constante la alusión a una nueva cultura de aprendizaje forjada en supuestos que, en ocasiones, pueden resultar poco operativos o difíciles de integrar en nuestro contexto más de corte academicista, como así reflejan algunos de los ítems del cuestionario utilizado.

No tanto porque se ponga en entredicho la eficacia práctica del nuevo enfoque, ya que se ajusta a los requerimientos del mercado laboral y social, sino que porque "se alejan" de lo acostumbrado en el aula: su implantación desdibuja las tareas tradicionales de profesores y alumnos frente al aprendizaje. En especial para los docentes, pues se les insta a una mayor entrega y disposición hacia al alumno así como el uso de nuevos métodos didácticos que, incluso, sorprenden. Son profesionales considerados, socialmente por su faceta de expertos en un área, más que por su domino pedagógico o didáctico. Sin embargo, los datos del ALCP se aproximan a los encontrados por la propia McCombs en el estudio que realizó en el año 2002 con 263 profesores, pero estos se adhieren más a los principios de la enseñanza centrada en el alumno, otorgando validez a este trabajo.

Antes de describir las dos principales conclusiones, mencionar ciertas limitaciones de estudio: la muestra de profesores no es representativa pues está configurada 
por aquellos que voluntariamente respondieron al cuestionario y, a su vez, mostraban interés y disposición por asistir a las sesiones de formación organizadas por el Servicio de Innovación Docente de la Universidad de Navarra. Los alumnos son aquellos que cursan las asignaturas impartidas por los docentes de la muestra.

Dos son las principales conclusiones: en primer lugar, que los profesores de este estudio, globalmente, mostraron compartir y estar de acuerdo con los principios de una ECAA (es cierto que eran profesionales con cierta vocación docente y ello puede sesgar los datos). Los datos del ALCP se aproximan a los encontrados por la propia McCombs en el estudio que realizó en el año 2002 con 263 profesores, pero estos se adhieren más a los principios de la enseñanza centrada en el alumno, otorgando validez a este trabajo.

En segundo lugar, que es reseñable la falta de confianza en la capacidad del alumno para dirigir su aprendizaje. Ante esta cuestión, nos planteamos ¿el aprendizaje autorregulado será preferible solamente en últimos años de grado y en el posgrado?

De esta forma, retoma protagonismo el trabajo colaborativo entre los profesores. No tiene sentido que ciertos profesores replanteen su enseñanza hacia tales premisas mientras otros sigan anclados exclusivamente en método tradicionales, pues los alumnos no se percatarán del valor que, para su futura formación, desempeña una enseñanza centrada en el aprendizaje.

Investigaciones futuras en este campo deberían replantear la introducción de las estrategias propias del aprendizaje autorregulado en los planes de estudio de los grados. Esto es, si el alumno asume cada vez un estudio más autónomo, conviene que adquiera esas herramientas que permiten tomar las riendas de su formación. Por otra parte, sería interesante profundizar en las características o modelo de los cursos o programas de formación docente en aras de lograr profesores cuya enseñanza se adecue a las exigencias del EEES. Esto es, el desarrollo profesional docente debería regirse por las mismas premisas del aprendizaje autorregulado demandado a los alumnos, así asumirían prácticas y ejemplos directos para sus estudiantes que podrán reproducir en el aula con mayor facilidad.

\section{REFERENCIAS BIBLIOGRÁFICAS}

BARDIN, L. (1986). Análisis de contenido. Madrid: Akal Universitaria.

BARELSON, B. (1952). Content analysis. En G. Lindzey (ed.), Handbook of Social Psychology (pp. 488-522). London: Adisson Wesley.

BERICAT, E. (1998). La integración de los métodos cuantitativo y cualitativo en la investigación social. Barcelona: Ariel.

CHOCARRO, E.; GONZÁLEZ-TORRES, M.C. y SOBRINO, A. (2007). Nuevas orientaciones en la formación del profesorado para una enseñanza centrada en la promoción del aprendizaje autorregulado de los alumnos. Estudios Sobre Educación, 12, 81-98.

CRESWELL, J.W. y PLANO CLARK, V.L. (2007). Designing and conducting mixed methods research. Thousand Oaks: SAGE Publications.

CRESWELL, J.W. (2009). Research Design: qualitative, quantitative and mixed methods approaches. Thousand Oaks: SAGE Publications. 
DE LA FUENTE, J.; PICHARDO, M.C.; JUSTICIA, F. y BERBÉN, A. (2008). Enfoques de aprendizaje, autorregulación y rendimiento en tres universidades europeas. Psicotema, 20 (4), 705-711.

ENTWISTLE, N.; SKINNER, D.; ENTWISTLE, D. y ORR, S. (2000). Conceptions and beliefs about "Good Teaching": an integrating of contrasting research areas. Higher Education Research and Development, 19 (1), 5-26.

FINK, D.L. (2003). Creating significant learning experiences: an integrated approach to designing college course. San Francisco: Jossey-Bass.

HATIVA, N. (1997). Teaching in a research university: proffesor's conceptions, practices and disciplinary differences. Comunicación presentada en Annual Meeting of the American Educational Research Association, Chicago.

HO, A. (2000). A conceptual change approach to staff development: a model for programme design. The International Journal for Academic Development, 5, 30-41.

MARCELO, C. (2002). Aprender a enseñar para la sociedad del conocimiento. Education Policy Analysis Archives, 10 (35), 1-52.

MCCOMBS, B. (1997). Self-assessment and reflection: tools for promoting teachers changes toward learners-centered practices. Instructional Strategies, 81, 1-14.

MCCOMBS, B. y PIERCE, J.W. (1999). Development and validation of the college level Assessment of Learner-Centered Practices (ALCP) surveys. Denver, CO: University of Denver. Inédito.

MCCOMBS, B. y WHISLER, J. (2000). La clase y la escuela centradas en el aprendiz. Barcelona: Paidós.

POSTAREFF, L.; LINDBLOM-YLÄNNE, S. y NEVGI, A. (2007). The effect of pedagogical training on teaching in higher education. Teaching and Teacher Education, 23 (5), 557-571.

POSTAREFF, L. y LINDBLOM-YLÄNNE, S. (2008). Variation in teachers' descriptions of teaching: broadening the understanding of teaching in higher education. Learning and Instruction, 18 (2), 109-120.

POZO, J.I. (2006). La nueva cultura del aprendizaje en la sociedad del conocimiento. En J.I. Pozo Municio y M. Scheuer (eds.), Nuevas formas de pensar la enseñanza y el aprendizaje. Las concepciones de profesores y alumnos (pp. 29-55). Barcelona: Graó.

POZO, J.I. (2008). Aprendices y maestros (2a ed.). Madrid: Alianza.

REIS-JORGE, J. (2007). Teacher's conceptions of teacher research and self-perceptions as enquiring practitioners. A longitudinal case study. Teaching and Teacher Education, 23, 402-417.

SALOMON, G. (1992). The changing role of the teacher: from information transmitter to orchestrator of learning. En K. Oser, A. Dick y J.L. Patry (eds.), Effective and responsible teaching (pp. 35-49). San Francisco: Jossey Bass.

SAMUELOWICZ, K. (1999). Academic's educational beliefs and teaching practices. Brisbane: Griffith University. Tesis doctoral inédita.

STES, A.; GIJBELS, D. y VAN PETERGEN, P. (2007). Student-focused approaches to teaching in relation to context and teacher characteristics. Comunicación presentada en American Educational Research Association, Chicago. 
TORRANO, F. y GONZÁLEZ-TORRES, M.C. (2004). El aprendizaje autorregulado: presente y futuro de la investigación. Revista Electrónica de Investigación Psicoeducativa, 2 (1), 1-34.

VAN DRIEL, J.; BULTE, A. y VERLOOP, N. (2007). The relationships between teachers' general beliefs about teaching and learning and their domain specific beliefs. Learning and Instruction, 17, 156-171.

WEIMER, M. (2002). Learned-centered teaching: five keys changes to practice. San Francisco: Jossey Bass. 